\title{
CmRCD1 represses flowering by directly interacting with CmBBX8 in summer chrysanthemum
}

\author{
Lijun Wang ${ }^{1,3}$, Hua Cheng ${ }^{1}$, Qi Wang ${ }^{1}$, Chaona Si ${ }^{1}$, Yiman Yang ${ }^{1}$, Yao Yu', Lijie Zhou' ${ }^{1}$ Lian Ding ${ }^{1}$, Aiping Song ${ }^{1}$, \\ Dongqing $\mathrm{Xu}^{2}$, Sumei Chen ${ }^{1}$, Weimin Fang ${ }^{1}$, Fadi Chen ${ }^{1}$ and Jiafu Jiang (id)
}

\begin{abstract}
The CMBBX8-CmFTL1 regulatory module is a key determinant in the transition from vegetative growth to reproductive development in summer-flowering chrysanthemum. However, the detailed regulatory mechanism of CmBBX8mediated flowering remains elusive. In this study, we revealed that RADICAL-INDUCED CELL DEATH 1 (CmRCD1) physically associated with $\mathrm{CmBBX8}$ through bimolecular fluorescence complementation (BiFC), pulldown and Coimmunoprecipitation (CoIP) assays. Furthermore, the RCD1-SRO1-TAF4 (RST) domain of CmRCD1 and the B-box of CmBBX8 mediated their interaction. In addition, Luciferase (LUC) assays and electrophoretic mobility shift assay (EMSAs) showed that $C m R C D 1$ repressed the transcriptional activity of $C m B B X 8$ and interfered with its binding to the CMFTL1 promoter, thereby leading to delayed flowering in the summer chrysanthemum 'Yuuka'. These results provide insight into the molecular framework of CmRCD1-CmBBX8-mediated flowering in chrysanthemum.
\end{abstract}

\section{Introduction}

Flowering is a major stage of the transition from vegetative to reproductive growth during the plant life cycle. Plants have evolved a precise regulatory network to initiate flowering in response to various external and internal cues. Extensive studies have documented that $\mathrm{BBX}$ proteins work in concert with other key components of flowering to mediate the initiation of flowering in various plant species. Arabidopsis BBX10 and BBX19 directly interact with CONSTANS $(\mathrm{CO})$ to repress the transcriptional activation of FLOWERING LOCUS T $(F T)$, which in turn leads to a reduction in $F T$ and FTcontrolled gene expression and then brings about a subsequent delay in flowering ${ }^{1,2}$. AtBBX4 directly associates with the $F T$ promoter in the presence of AtBBX32 to

\footnotetext{
Correspondence: Jiafu Jiang (jiangjiafu@njau.edu.cn)

${ }^{1}$ State Key Laboratory of Crop Genetics and Germplasm Enhancement, Key Laboratory of Landscaping, Ministry of Agriculture and Rural Affairs, Key Laboratory of Biology of Ornamental Plants in East China, National Forestry and Grassland Administration, College of Horticulture, Nanjing Agricultural University, Nanjing 210095, China

${ }^{2}$ State Key Laboratory of Crop Genetics and Germplasm Enhancement, College of Agriculture, Nanjing Agricultural University, Nanjing 210095, China Full list of author information is available at the end of the article
}

repress its transcription ${ }^{3}$. The CO-FKBP12 interaction contributes to the modulation of photoperiodic flowering, resulting in a decrease in $F T$ in the early morning ${ }^{4}$. In addition, both $\mathrm{BBX} 30$ and $\mathrm{BBX} 31$ recruit $\mathrm{CO}$ into a TOPLESS trimeric complex to inhibit $F T$ expression and flowering $^{5}$. In chrysanthemum, multiple BBX proteins mediate flowering, either negatively or positively, through distinct regulatory mechanisms. CmBBX8, a member of the BBX family containing a CCT domain, directly associates with the promoter regions of FTL1 to activate its transcription and accelerate flowering ${ }^{6}$. $\mathrm{CmBBX} 24$ and CmBBX13 negatively control flowering by repressing the expression of flowering-promoting factors ${ }^{7,8}$.

RCD1 is a member of the poly-ADP ribose polymerase (PARP) family ${ }^{9,10}$. Mutants with loss of RCD1 function are hypersensitive to reactive oxygen species (ROS) and exhibit early flowering ${ }^{11-14}$. The WWE domain within RCD1 functions as a specific protein-protein interaction motif $^{15}$, and the PARP catalytic domain within RCD1 contains multiple conserved residues that are required for the formation of donor sites (Gly-347, Leu-348, Ser-375) or acceptor sites (Tyr-378). These distinct functional residues contribute to diverse cellular processes, including

\section{(c) The Author(s) 2021}

\footnotetext{
(cc) Open Access This article is licensed under a Creative Commons Attribution 4.0 International License, which permits use, sharing, adaptation, distribution and reproduction in any medium or format, as long as you give appropriate credit to the original author(s) and the source, provide a link to the Creative Commons license, and indicate if changes were made. The images or other third party material in this article are included in the article's Creative Commons license, unless indicated otherwise in a credit line to the material. If material is not included in the article's Creative Commons license and your intended use is not permitted by statutory regulation or exceeds the permitted use, you will need to obtain permission directly from the copyright holder. To view a copy of this license, visit http://creativecommons.org/licenses/by/4.0/.
} 
chromatin remodeling, genomic imprinting, and transcriptional regulation ${ }^{14,16-18}$. The RST domain present in RCD1 is involved in the assembly of the multimeric general transcription factor IID complex (TFIID) $)^{13,19}$. Although it has been demonstrated that RCD1 associates with the BBX protein from Arabidopsis in yeast, the biological consequence to flowering of this association remains elusive ${ }^{13}$.

In this study, we report that CmRCD1 interacts with CmBBX8 to repress the expression of CmFTL1, thereby resulting in inhibition of flowering in cv. 'Yuuka'. Collectively, our results reveal a regulatory module consisting of CmRCD1, CmBBX8 and CmFTL1 in the regulation of flowering.

\section{Results}

\section{Isolation and expression pattern of $C m R C D 1$}

To investigate the biological functions of CmRCD1 in chrysanthemum, a CmRCD1 sequence was isolated from 'Yuuka' with primers designed in Primer 5.0 that amplified a $1728 \mathrm{bp}$ open-reading frame (ORF) predicted to encode a 575-residue polypeptide consisting of the PARP (amino acids 313-438) and RST (amino acids 510-575) domains (Fig. 1a). Phylogenetic analysis confirmed a close relationship between the 'Yuuka' RCD1 and Artemisia апnиа L. RCD1 (Fig. 1b) after amino acid alignment (Fig. S1). Because CmBBX8 has been found to exhibit diurnally controlled expression in our previous research ${ }^{6}$ and because CmRCD1 may interact with $\mathrm{CmBBX} 8$, the transcript levels of $C m R C D 1$ under the regulation of a diurnal clock were further investigated. RNA was extracted from 'Yuuka' leaves, and the plants were grown for 2 weeks under long-day (LD) conditions, short-day (SD) conditions, continuous illumination (LL) conditions or continuous darkness (DD) conditions for $48 \mathrm{~h}$. The results showed that the expression of $C m R C D 1$ was not controlled by photoperiod rhythm (Fig. S2).

\section{CmRCD1 interacted with CmBBX8}

Next, the subcellular localization of CmRCD1 was investigated by transiently expressing the gene in tobacco. Green fluorescent protein (GFP) activity was observed in both the cytoplasm and the nucleus in tobacco cells transiently transformed with the p35S:: GFP control plasmid (Fig. S3). The GFP signal overlapped with that of the nuclear marker D53-mCherry in tobacco cells when CmRCD1 was transiently expressed and fused with GFP driven by the CaMV35S promoter (Fig. S3), suggesting that CmRCD1 may localize in the nucleus. A BiFC assay was used to detect the interaction between $\mathrm{CmRCD} 1$ and $\mathrm{CmBBX} 8$ in tobacco leaves; Yellow fluorescent protein (YFP) activity and the nuclear marker overlapped differently in the experimental plasmids than for the empty vector (EV) control (Fig. 2a). The interaction between CmRCD1 and CmBBX8 was further verified using a pulldown assay. His-CmBBX8, a GST EV and GST-CmRCD1 were all detected as input: the GST-fusion proteins were detected using anti-GST, and the His-fusion proteins were detected using anti-His. The results indicated that these proteins were present in the assay (Fig. 2b). The signal of GST protein with the His-fusion CmBBX8 protein was used as a negative control. Only the HisCmBBX8 protein was immunodetected using anti-His when GST-RCD1 was used as bait in the pulldown assay. The GST empty protein had no His-CmBBX8 protein signal. These results showed that GSTCmRCD1 alone pulled down His-CmBBX8 (Fig. 2b). Moreover, Flag-CmRCD1 was immunoprecipitated by HA-CmBBX8 when transiently coexpressed in tobacco

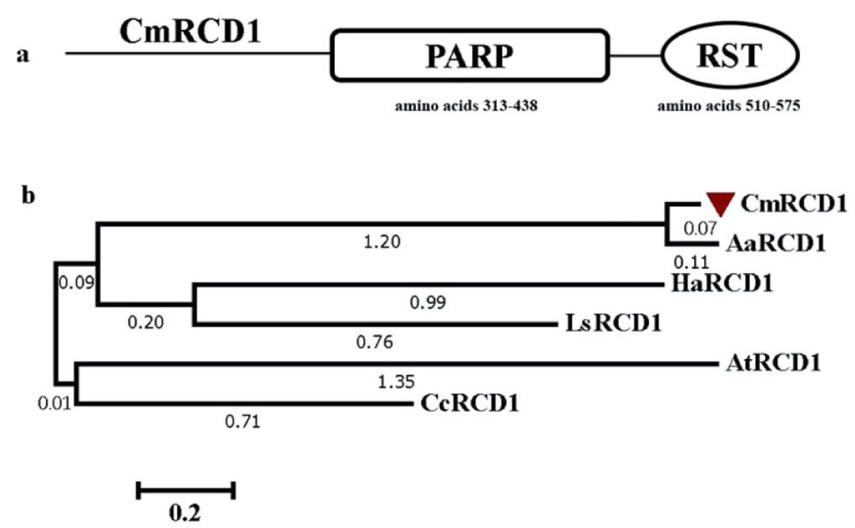

Fig. 1 Structure and evolutionary tree of the CmRCD1 protein. a Predicted protein structure. $\mathbf{b}$ Evolutionary tree of RCD1 in chrysanthemum and other plant species. AaRCD1: Artemisia annua RCD1 (PWA95938.1); HaRCD1: Helianthus annuus RCD1 (XP_021969868.1); LsRCD1: Lactuca sativa RCD1 (XP_023736213.1); AtRCD1: Arabidopsis thaliana RCD1 (AT1G32230.1); CcRCD1: Cynara cardunculus RCD1 (XP_024993358.1) 


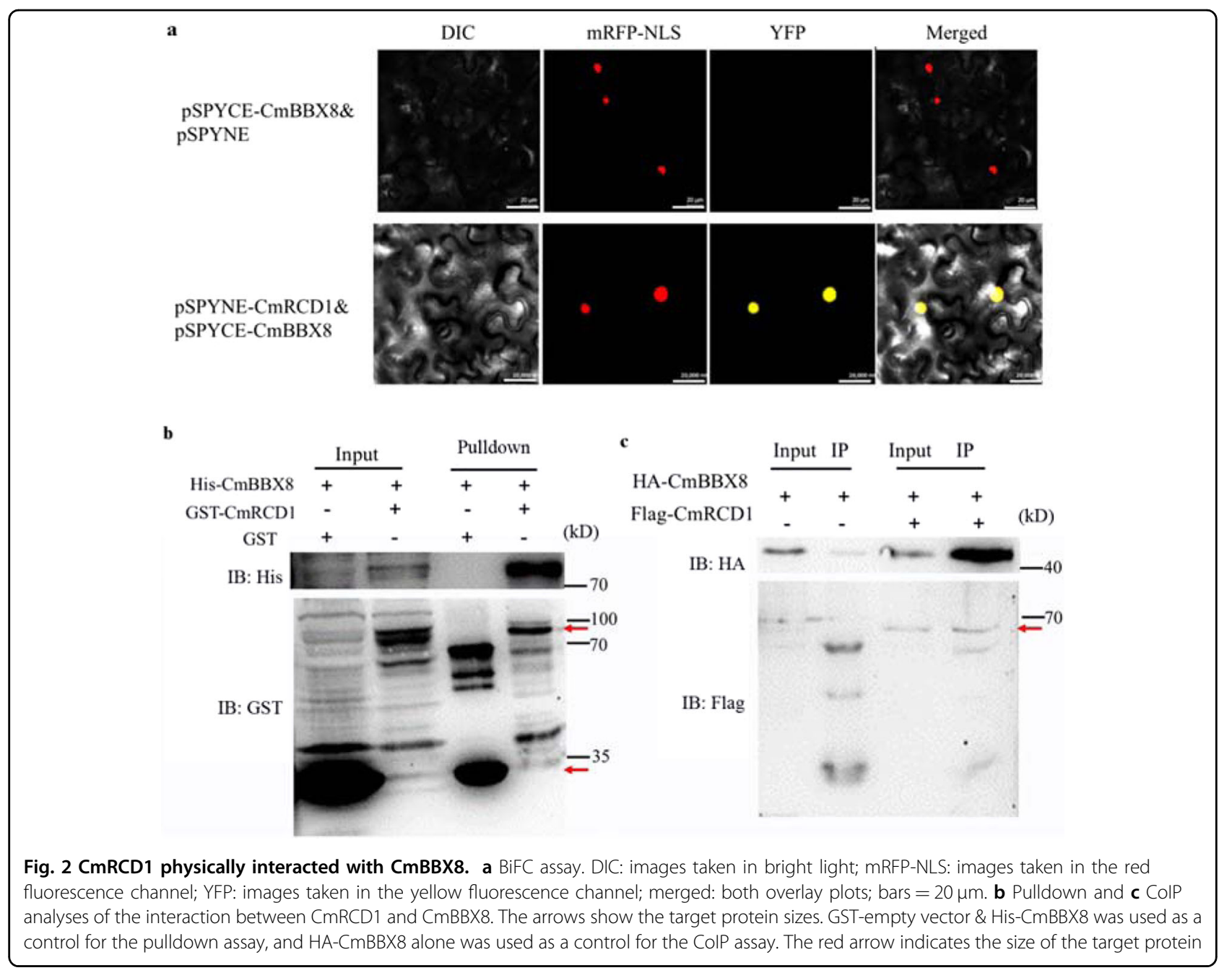

leaf cells (Fig. 2c). Together, these results suggest that CmRCD1 interacts with CmBBX8 both in vivo and in vitro.

\section{CmRCD1 interacted with the B-box domain of CmBBX8 through its RST domain}

Homology modeling analysis using AtRCD1 as a template on the Swiss-Model website revealed that the CmRCD1 protein lacked PARP catalytic activity due to the loss of the Gly-347, Leu-348, and Ser-375 donor sites (Fig. S4). These data indicated that the PARP domain was not involved in the catalysis of CmBBX8. In a yeast two-hybrid assay, when different domains of RCD1 were truncated, the RST domain of $\mathrm{CmRCD} 1$ was found to physically interact with the B-box domain of CmBBX8: the yeast grew well on $\mathrm{SD} /$ $\mathrm{Trp}^{-} \mathrm{Leu}^{-} \mathrm{His}^{-} \mathrm{Ade}^{-}$medium and exhibited blue coloration on $\mathrm{SD} / \mathrm{Trp}^{-} \mathrm{Leu}^{-} \mathrm{His}^{-} \mathrm{Ade}^{-}$medium containing X- $\alpha$-Gal (Fig. 3a). Therefore, the RST domain was shown to be the key structure of the interaction (Figs. S3b and S5).

\section{CmRCD1 associated with $\mathrm{CmBBX8}$ to repress the upregulation of CMFTL1}

CmBBX8 acts as a flowering inducer to regulate the expression of $C m F T L 1^{6}$. To clarify the function of CmRCD1 in the pathway, reporter and effector vectors were constructed (Fig. 4a). A LUC detection assay of tobacco was used to characterize the effects of CmRCD1 and CmBBX8 on the CmFTL1 promoter based on a previously described method ${ }^{20}$. Compared with the empty vector $(\mathrm{EV})$, the $\mathrm{CmBBX} 8$ protein was more capable of activating LUC driven by CmFTL1 promoter, while the opposite results were observed for the CmRCD1 protein, and the presence of $\mathrm{CmRCD} 1$ reduced the activating effect of CmBBX8 (Fig. 4b). To confirm the above results, Renilla luciferase (REN) was used as an internal reference according to a previously described method ${ }^{20}$, and an Arabidopsis protoplast transfection experiment showed that $\mathrm{CmRCD} 1$ could indeed significantly reduce the ability of CmBBX8 to activate LUC under the control of the 
a

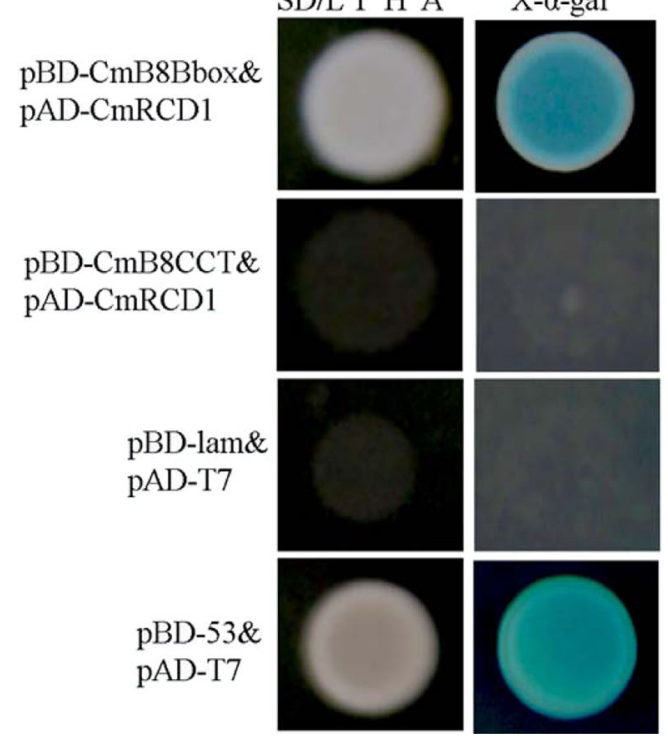

b

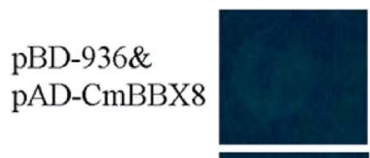

pBD-PARP\& pAD-CmBBX8

pBD-RST\& pAD-CmBBX8
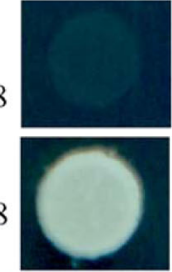

pBD-lam\& pAD-T7

pBD-53\& pAD-T7
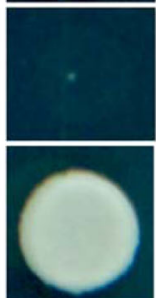


Fig. 3 The RST domain of CmRCD1 and the B-box domain of CmBBX8 were required for the CmRCD1-CmBBX8 interaction in yeast cells. p53\&pT7 and pT7\&plam were used as the positive and negative controls, respectively. a Interaction between the segments of $\mathrm{CmBBX} 8$ and CmRCD1. $\mathbf{b}$ Interaction between the segments of CmRCD1 and CmBBX8
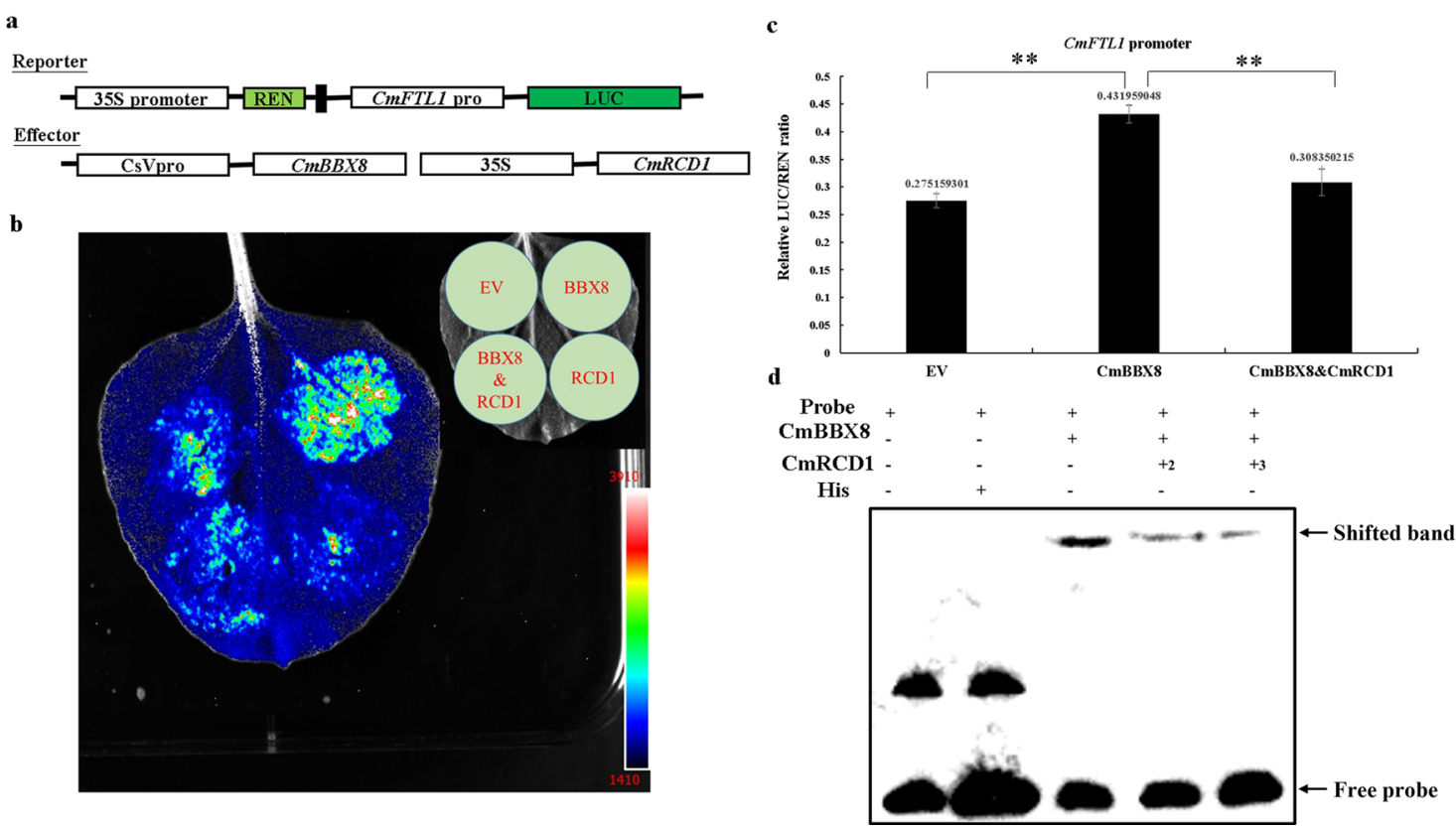

Fig. 4 LUC assays and EMSAs verified the mechanism of interaction between CmBBX8 and CmRCD1. a Structure of the vector. b Antagonistic effects of CMRCD1 on CMBBX8 activation of the CMFTL1 promoter; from blue to red, the fluorescence value increases gradually. c Relative LUC/REN ratios in Arabidopsis protoplasts. Student's $t$ test was employed; ${ }^{* *}$ indicates a highly significant difference $(P<0.01)$, and the error bars indicate the SEs $(n=3)$. $\mathbf{d}$ EMSA results. The two left lanes represent the free combination reactions with the biotin-labeled probe and the His-tag with the biotinlabeled probe, the third lane represents the $\mathrm{CmBBX} 8$ protein with the biotin-labeled probe, the fourth lane represents the $\mathrm{CmBBX} 8$ protein with the biotin-labeled probe and $2 \mu \mathrm{L}$ of $\mathrm{CmRCD} 1$ protein, and the fifth lane represents the $\mathrm{CmBBX} 8$ protein with the biotin-labeled probe and $3 \mu \mathrm{L}$ of CmRCD1 protein 

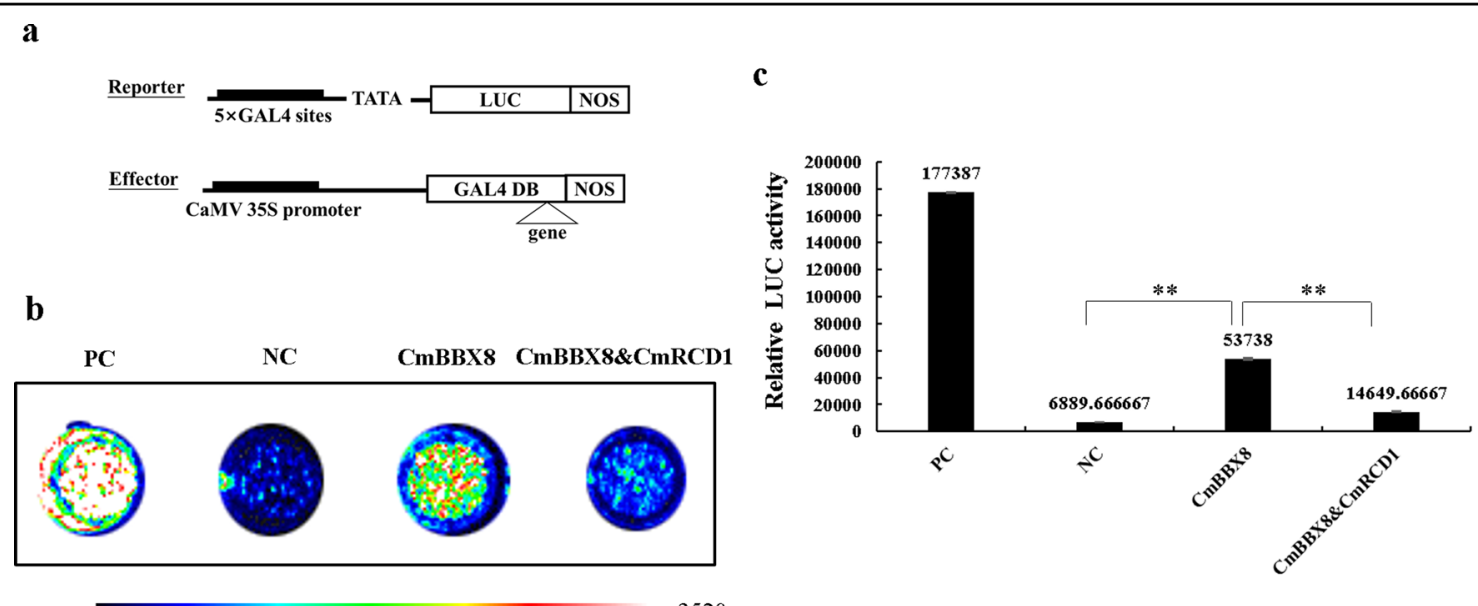

Fig. 5 CmRCD1 repressed CmBBX8 activity. PC: positive control (AtARF5); NC: negative control (empty vector). a Structure of the vector. $\mathbf{b}$ Fluorescence images; from blue to red, the fluorescence value increases gradually. c Relative LUC activity. The error bars indicate the SEs $(n=3)$; **represents a highly significant difference (Student's $t$ test) $(P<0.01)$

CmFTL1 promoter (Fig. 4c). In addition, an EMSA was used to characterize the DNA-protein interactions involving $\mathrm{CmBBX} 8$ and $\mathrm{CmRCD} 1$. Consistent with the findings of a previous study ${ }^{6}$, the CCT domain of CmBBX8 was able to bind to the CmFTL1 promoter subfragment. The binding ability of $\mathrm{CmBBX} 8$ protein was decreased in the presence of CmRCD1 protein. As the amount of CmRCD1 increased in the reaction, the CmBBX8-binding affinity decreased (Fig. 4d). Overall, these results indicate that CmRCD1 interferes with CmBBX8 binding to the CmFTL1 promoter.

As the RST domain participates in the assembly of the multimeric general transcription factor complex TFIID $^{19}$, we investigated whether CmRCD1 affected the transcriptional activation function of CmBBX8. An Arabidopsis protoplast transfection experiment showed that the transcriptional activation function of CmBBX8 was inhibited by CmRCD1 (Fig. 5a, b). In addition, the relative LUC activity of CmRCD1's participation was significantly reduced when CmBBX8 was co-expressed (Fig. 5c). Therefore, these data indicate that CmRCD1 also negatively affected the transcriptional activation function of CmBBX8 through its RST domain, thereby repressing the expression of CmFTL1.

\section{CmRCD1 delayed flowering in chrysanthemum}

To determine whether CmRCD1 participates in the genetic regulation of flowering time, $C m R C D 1$ was specifically knocked down using an artificial microRNA ${ }^{21}$. The amiR-CmRCD1 plants initiated their first involucral primordia 45 days after transplanting, while initiation of the primordia of WT (Wild Type) plants was delayed by approximately one week (Fig. 6a, b). Furthermore, CmFTL1 was upregulated in amiR-CmRCD1 transgenic plants (Fig. 6c). These data indicate that $C m R C D 1$ delayed flowering through downregulation of CmFTL1.

\section{Discussion}

$\mathrm{CO} / \mathrm{BBX} 1$ is a key component of photoperiodic flowering in Arabidopsis ${ }^{22}$. In addition to CO/BBX1, multiple other BBX proteins have been shown to play critical roles in the flowering of different plant species. Here, we revealed that $\mathrm{CmRCD} 1$ formed inactive heterodimers with CmBBX8, which is a direct activator of CmFTL1. The CmRCD1-CmBBX8 regulatory module modulated CmFTL1 transcription by fine-tuning the initiation of flowering in chrysanthemum.

Arabidopsis RCD1 possesses a WWE domain, a PARPlike (poly [ADP-ribose] polymerase-like) domain, and an RST domain at the C-terminal region ${ }^{14}$. RCD1 plays pleiotropic roles in various developmental processes and in response to diverse abiotic and biotic stresses in plants $^{13,21,23}$. A yeast two-hybrid assay revealed that RCD1 interacts with a large number of transcription factors, such as ethylene responsive factors (AP2/ERF), NAC family transcription factors (NAM, ATAF1/2 and CUC2) and basic Helix-Loop-Helix (bHLH) transcription factors ${ }^{13}$. A growing number of studies have shown that RCD1 interacts with the transcription factors ANAC013 ${ }^{24}, \mathrm{DREB}_{2 \mathrm{~A}^{25}}$ and Rap2.4a ${ }^{26}$, and affects their transcriptional activation function. These facts suggest that RCD1 functions as a transcriptional regulator by directly associating with multiple transcription factors and affecting their behavior. Via yeast assays, an interaction between RCD1 and COL9 (BBX7) has been found, but the mechanism is unknown ${ }^{13}$. CmBBX8 directly binds to the promoters of CmFTL1 to activate its expression, thereby promoting flowering ${ }^{6}$. Here, we found that 


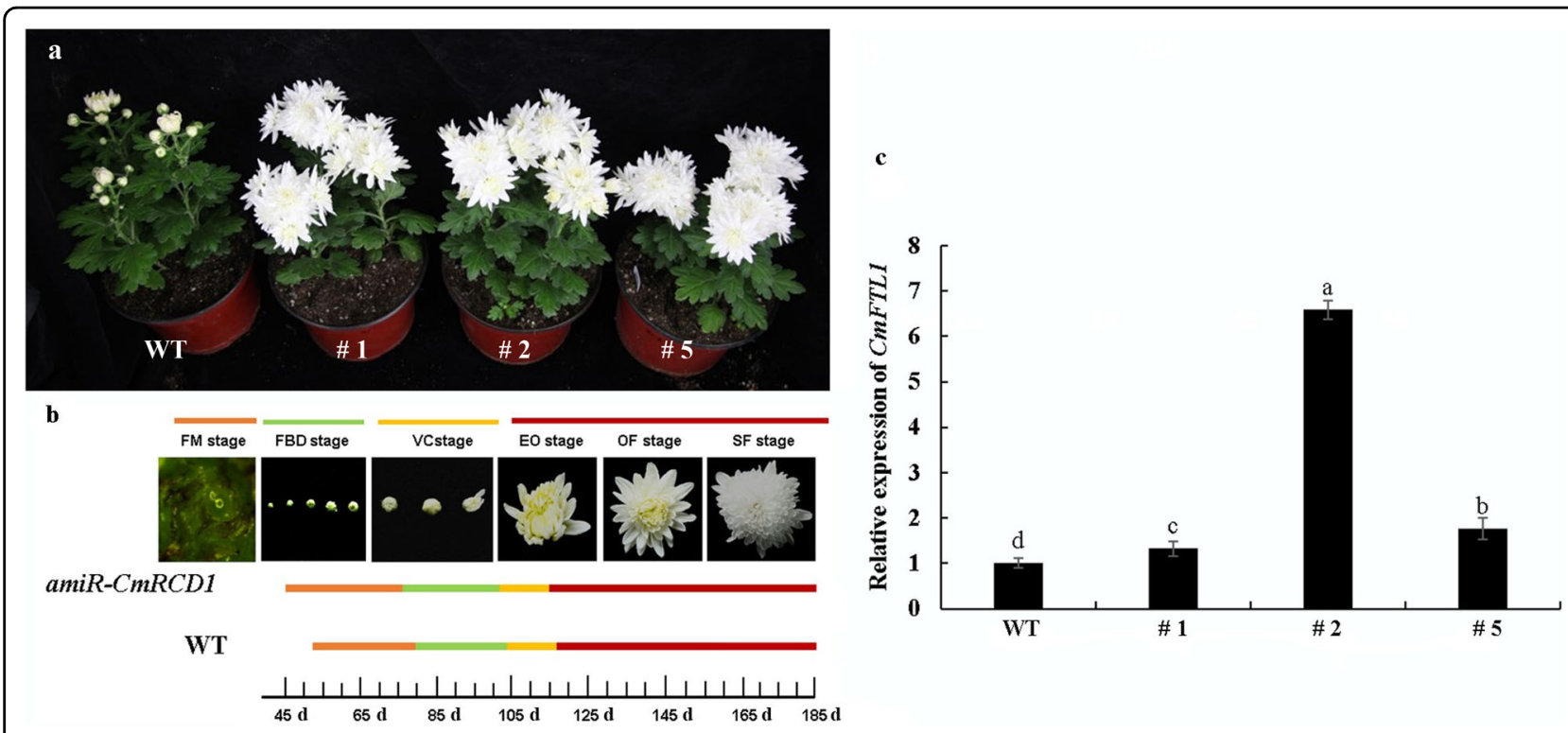

Fig. 6 Phenotype of amiR-CmRCD1 transgenic 'Yuuka' at the flowering stage. Wild type: $W T ; \# 1, \# 2$, \#5: amiRNA plants. a Flowering phenotype. b Statistics of flowering time. FM: Floral meristem stage; FBD: Flower bud development stage; VC: Visible color stage; EO: Early opening stage; OF: Opened flower stage; SF: Senescing flower stage. c Relative expression of CmFTL1 in amiR-CMRCD1 plants. Tukey's honestly significant difference (HSD) test was employed. Different letters above the bars indicate significant differences $(P<0.05)$; the error bars indicate the SEs $(n=3)$

CmRCD1 negatively controlled flowering via a similar molecular mechanism: CmRCD1 physically interacted with CmBBX8 to affect its biochemical activity, consequently inhibiting the expression of CmFTL1 and flowering in chrysanthemum (Figs. 4 and 5).

BBX protein-mediated floral initiation is a central theme of light-dependent plant development. CO-FT represents a key regulatory hub of flowering control, and this module is evolutionally conserved in various plant species $^{27,28}$. CO associates with the CORE ciselement present in the $F T$ promoter region to upregulate its expression, enabling the accumulation of FT. Accumulated foreign FT in the leaves shifts to the shoot apex, where it initiates floral development ${ }^{29}$. CO, which is a B-box-containing protein, directly controls FT at the transcriptional level. To maintain a proper FT level, a set of factors converge on $\mathrm{CO}$ to modulate its activity. For instance, BBX10, BBX19 and TOEs mediate CO activity through direct protein-protein interactions ${ }^{1,2,30}$; this appears to be a common regulatory mode for control of $\mathrm{CO}$ activity.

The function of the CmBBX8-CmFTL1 module is critical for initiation of flowering in chrysanthemum. Our previous studies have shown that $\mathrm{CmBBX} 8$ directly associates with the CORE cis-element (CCACA) within the CmFTL1 promoter and activates its transcription. CmFTL1, a close ortholog of FT, accelerates flowering in chrysanthemum grown under LD conditions. CmRCD1 negatively controls the initiation of flowering through the CmBBX8-CmFTL1-mediated pathway and represses the binding of CmBBX8 to the CmFTL1 promoter by directly interacting with CmBBX8. These molecular events consequently lead to inhibition of CmFTL1 at the mRNA level and to repression of flowering in chrysanthemum. Thus, the CmRCD1-CmBBX8-CmFTL1 signaling pathway may precisely control flowering in chrysanthemum in response to dynamically changing environmental conditions, which is potentially relevant for chrysanthemum molecular breeding strategies.

\section{Materials and methods}

Isolation of CmRCD1 and analysis of its structure

The CmRCD1 ORF sequence was amplified using the primer pair CmRCD1-F/-R (annexed table, Wang et al., $2020^{21}$ ). The conserved domains were analyzed using the NCBI database (www.ncbi.nlm.nih.gov/Structure/cdd/wrpsb. cgi). The phylogeny of a set of RCD1 sequences recovered from GenBank was derived using MEGA7.0 software with the neighbor-joining algorithm and $1000 \mathrm{bp}$ replications ${ }^{31}$.

\section{Yeast two-hybrid experiments}

To introduce the pENTR1A-CmBBX8 construct, the primer set CmBBX8 (BamH I)-F/CmBBX8 (EcoR I)-R (annexed table) was used and recombined into the final pGADT7 vector using the LR recombination reaction. The CmBBX8-Bbox and CCT segment sequences were amplified using primer pairs designed to incorporate an EcoR I site at one end of the amplicon and a BamH I site at the other end (annexed table). BamH I/EcoR I digestion and ligation of the pGBKT7 and amplified segments were 
then performed. The CmRCD1-PARP, RST and 936segment sequences were amplified by using primer pairs designed with the CE Design app (annexed table) and by subsequently conducting a separate homologous recombination reaction with pGADT7. The resulting constructs were transferred into the yeast strain $Y 2 H$. Yeast cells were cultured on synthetic dropout (SD)/Leu ${ }^{-} \mathrm{Trp}^{-}$medium for 3 days at $30^{\circ} \mathrm{C}$ and then transferred to $\mathrm{SD} /$ $\mathrm{His}^{-} \mathrm{Ade}^{-} \mathrm{Leu}^{-} \mathrm{Trp}^{-}$plates in either the presence or absence of X- $\alpha-G a l$.

\section{Bimolecular fluorescence complementation assay and luciferase assay}

The $C m R C D 1$ and $C m B B X 8$ sequences were amplified using the primer pairs CmBBX8 YFP-F/CmBBX8 YFP-R and CmRCD1 YFP-F/CmRCD1 YFP-R (annexed table) with $B a m \mathrm{H}$ I and Kpn I and were inserted into the pSPYNE/YCE vector, which contains a reporter gene encoding $\mathrm{YFP}^{32}$. The A. tumefaciens strain GV3101 harboring CmBBX8 and CmRCD1 was grown to an $\mathrm{OD}_{600}$ of 0.5 , and infiltration medium (10 mM MES, $200 \mu \mathrm{M}$ AS, $10 \mathrm{mM} \mathrm{MgCl}_{2}$ ) was introduced via syringe into the leaves of a 5-6-week-old Nicotiana benthamiana plant. After 48-96 h, a confocal laser scanning microscope (ZEISS, LSM780) was used to observe the YFP signal according to a previously described method $^{33}$, and a CCD camera (Tanon 5200) was used to observe luciferase activity following a published method ${ }^{34}$.

\section{Pulldown assay}

To introduce the pENTR1A-CmRCD1 construct, the primer set CmBBX8 (BamH I)-F/CmBBX8 (EcoR I)-R (annexed table) was used and recombined into the final vector pDEST-15 (GST tag) using the LR recombination reaction. pDEST-15-CmRCD1, together with His-CmBBX8 (Kpn I/Pst I) (annexed table) constructed previously, was transfected into BL21 E. coli to induce protein expression. Next, $20 \mu \mathrm{L}$ of prewashed GST magnetic beads (Promega, Wisconsin-Madison) were added to the protein-containing supernatant and incubated at $4^{\circ} \mathrm{C}$ overnight. WB detection was performed with a His antibody (Thermo, USA) ${ }^{35}$.

\section{Coimmunoprecipitation assay}

pCsVMV-HA3-N-1300-CmBBX8 was constructed in a homologous recombination reaction, and pENTR1ACmRCD1 was recombined into the final vector pEarleyGate202-CmRCD1 (Flag tag) using the LR recombination reaction. The A. tumefaciens strain GV3101 harboring the constructs pEarleyGate202-CmRCD1 and pCsVMV-HA3-N-1300-CmBBX8 was introduced into $N$. benthamiana plants. After 48-96 h, the leaves were sampled, and proteins were extracted with EB buffer $(100 \mathrm{~mL}$ :

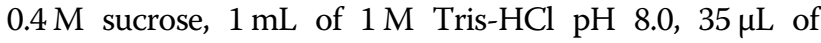
$14.3 \mathrm{M}$ stock $\beta$-Me, $100 \mu \mathrm{L}$ of $100 \mathrm{mM}$ PMSF, 2 cocktail tablets). The protein was first incubated with $20 \mu \mathrm{L}$ of $\mathrm{HA}$ beads at $4^{\circ} \mathrm{C}$ overnight (Thermo, USA) and then incubated with a Flag antibody for WB detection ${ }^{36}$.

\section{Transient activation experiments in protoplasts}

GALDB4-CmRCD1 and GALDB4-CmBBX8 were constructed according to the above LR recombination method. Protoplasts were prepared from 3-week-old Columbia WT Arabidopsis plants by affixing the epidermal cells with scotch tape, immersing them in an enzymolysis solution (20 mL: $1 \mathrm{~mL}$ of $100 \mathrm{mM}$ MES, 1.44 $\mathrm{g}$ of D-mannitol, $0.2 \mathrm{~g}$ of cellulase R10, and $0.02 \mathrm{~g}$ of pectinase $\mathrm{R} 10$ ) at $55^{\circ} \mathrm{C}$ for $10 \mathrm{~min}$, and then cooling the solution to $25^{\circ} \mathrm{C}$. Next, $1.6 \mathrm{~mL}$ of $100 \mathrm{mM} \mathrm{CaCl}_{2}$ and $0.03 \mathrm{~g}$ of BSA were added at $28^{\circ} \mathrm{C}$, and enzymatic hydrolysis was conducted for $1.5-2 \mathrm{~h}$ at $50 \mathrm{rpm}$ until the tape became transparent. Then, the 35mesh nylon membrane was rinsed with W5 buffer $(100 \mathrm{~mL}$ : $0.9 \mathrm{~g}$ of $\mathrm{NaCl}, 1.84 \mathrm{~g}$ of $\mathrm{CaCl}_{2}, 5 \mathrm{~mL}$ of $100 \mathrm{mM} \mathrm{KCl}$, and $2 \mathrm{~mL}$ of $100 \mathrm{mM} \mathrm{MES}$ ), and the protoplasts were filtered under a microscope. The protoplasts were then slowly washed with W5, and $1 \mathrm{~mL}$ of MMg buffer $(10 \mathrm{~mL}: 0.729 \mathrm{~g}$ of mannitol, $1.5 \mathrm{~mL}$ of $100 \mathrm{mM} \mathrm{MgCl} 2,400 \mu \mathrm{L}$ of $100 \mathrm{mM}$ MES) was gently added. After immersion in an ice bath for $30 \mathrm{~min}, 10 \mu \mathrm{g}$ of plasmids were transfected into $100 \mu \mathrm{L}$ of protoplasts with $110 \mu \mathrm{L}$ of PEG and W5 to stop the reaction $^{37}$. The solution was incubated under light for $16 \mathrm{~h}$, and $7.8 \mathrm{mM}$ sodium fluorescein was added. The relative LUC and REN activity was measured with a GloMax (GloMax ${ }^{\circledR}$ 20/20), and photographs were taken with a CCD camera (Tanon 5200).

\section{EMSA}

Protein expression and biotin probe application were conducted according to the protocols of the pulldown assay and Wang et al. $(2020)^{6}$, respectively. The subsequent EMSAs were performed using a Light Shift ${ }^{\mathrm{TM}}$ Chemiluminescent EMSA Kit (Thermo Fisher, New York) following the manufacturer's protocol ${ }^{6}$.

\section{qRT-PCR analysis}

RNA extraction, reverse transcription, and qRT-PCR were conducted following the manufacturer's protocol ${ }^{6}$. The primer pairs (qCmRCD1-F/qCmRCD1-R, qCmFTL1F/qCmFTL1-R) used to amplify CmBBX8 and CmRCD1 cDNA are given in the annexed table. Estimates of transcript abundance were calculated using the method published by Livak and Schmittgen ${ }^{38}$. The reference sequence was chrysanthemum EF1a (KF305681.1). Tukey's honestly significant difference test and Student's $t$ test were employed; a difference was considered significant at $P<$ 0.05 or $P<0.01$ for all data. For rhythm expression analysis, before the plants had formed 15-19 fully expanded leaves, they were held for 2 weeks under LD conditions. The plants were then transplanted into a cabinet supplying a 16 or $8 \mathrm{~h}$ photoperiod with continuous illumination and 
DD conditions. RNA was extracted from the third leaf from the shoot tip of cv. 'Yuuka'.

\section{Quantification of flowering time}

A microscope was used to recognize when chrysanthemum had entered the flowering transition stage and EB stage, at which point at least $50 \%$ of the ray flowers had unfolded $^{39}$. Statistics were tracked for the following periods: the FBD stage, VC stage, EO stage, OF stage, and SF stage. Measurements were taken from at least 20 plants $^{7}$.

\section{D homology modeling}

The catalytic activity of CmRCD1 was analyzed in the Swiss-Model database (https://www.swissmodel.expasy. org/) with AtRCD1 as the homology template.

\section{Acknowledgements}

We would like to thank Dr. Bo Sun from Nanjing University for helping with the ColP assay. This work was supported by the National Natural Science Foundation of China $(31930100,31872146)$ and a project funded by the Priority Academic Program Development of Jiangsu Higher Education Institutions.

\section{Author details \\ 'State Key Laboratory of Crop Genetics and Germplasm Enhancement, Key Laboratory of Landscaping, Ministry of Agriculture and Rural Affairs, Key Laboratory of Biology of Ornamental Plants in East China, National Forestry and Grassland Administration, College of Horticulture, Nanjing Agricultural University, Nanjing 210095, China. ${ }^{2}$ State Key Laboratory of Crop Genetics and Germplasm Enhancement, College of Agriculture, Nanjing Agricultural University, Nanjing 210095, China. ${ }^{3}$ Present address: College of Landscape and Ecological Engineering, Hebei University of Engineering, Handan 056038, China}

\section{Author contributions}

J.-F.J. and L.J.W. designed the experiments. L.-J.W. performed the experiments. L.-J.W., H.C., Q.W., C.-N.S., Y.Y., Y.-M.Y., L.-J.Z., L.D., A.P.S., and J.-F.J. analyzed the data. D.-Q.X., S.-M.C., W.-M.F., F.C. and J.-F.J. guided the research. J.-F.J., D.-Q.X. and L.J.W. wrote and revised the manuscript.

\section{Conflict of interest}

The authors declare no competing interests.

Supplementary information The online version contains supplementary material available at https://doi.org/10.1038/s41438-021-00516-z.

Received: 19 July 2020 Revised: 21 December 2020 Accepted: 6 February 2021

Published online: 01 April 2021

\section{References}

1. Wang, C., Guthrie, C., Sarmast, M. K. \& Dehesh, K. BBX19 interacts with CONSTANS to repress FLOWERING LOCUS T transcription defining a flowering time checkpoint in Arabidopsis. Plant Cell 26, 3589-3602 (2014).

2. Ordoñez-Herrera, $\mathrm{N}$. et al. The transcription factor COL12 is a substrate of the COP1/SPA E3 ligase and regulates flowering time and plant architecture. Plant Physiol. 176, 1327-1340 (2018).

3. Tripathi, P., Carvallo, M., Hamilton, E. E., Preuss, S. \& Kay, S. A. Arabidopsis BBOX32 interacts with CONSTANS-LIKE3 to regulate flowering. Proc. Natl Acad. Sci. USA 114, 172-177 (2017).

4. Serrano-Bueno, G. et al. CONSTANS-FKBP12 interaction contributes to modulation of photoperiodic flowering in Arabidopsis. Plant J. 101, 1287-1302 (2020).
5. Graeff, M. et al. Micro protein-mediated recruitment of CONSTANS into a TOPLESS trimeric complex represses flowering in Arabidopsis. PLoS Genet. 12, e1005959-e1005959 (2016).

6. Wang, L. et al. CmBBX8 accelerates flowering by targeting CMFTL1 directly in summer chrysanthemum. Plant Biotechnol. J. 18, 1562-1572 (2020).

7. Yang, Y. et al. A zinc finger protein regulates flowering time and abiotic stress tolerance in Chrysanthemum by modulating gibberellin biosynthesis. Plant Cell 26, 2038-2054 (2014).

8. Ping, Q. et al. The heterologous expression in Arabidopsis thaliana of a chrysanthemum gene encoding the BBX family transcription factor CmBBX13 delays flowering. Plant Physiol. Biochem. 144, 480-487 (2019).

9. Belles-Boix, E., Babiychuk, E., Van Montagu, M., Inzé, D. \& Kushnir, S. CEO1 a new protein from Arabidopsis thaliana protects yeast against oxidative damage. FEBS Lett. 482, 19-24 (2000).

10. D'Amours, D., Desnoyers, S., D'Silva, I. \& Poirier, G. G. Poly (ADP-ribosyl) ation reactions in the regulation of nuclear functions. Biochem. J. 342, 249-268 (1999).

11. Overmyer, K. et al. Ozone-sensitive arabidopsis rcd1 mutant reveals opposite roles for ethylene and jasmonate signaling pathways in regulating superoxidedependent cell death. Plant Cell 12, 1849-1862 (2000).

12. Ahlfors, R., Brosché, M., Kollist, H. \& Kangasjärvi, J. Nitric oxide modulates ozoneinduced cell death, hormone biosynthesis and gene expression in Arabidopsis thaliana. Plant J. 58, 1-12 (2009).

13. Jaspers, P. et al. Unequally redundant RCD1 and SRO1 mediate stress and developmental responses and interact with transcription factors. Plant J. 60, 268-279 (2009)

14. Teotia, S. \& Lamb, R. S. The paralogous genes RADICAL-INDUCED CELL DEATH1 and SIMILAR TO RCD ONE1 have partially redundant functions during Arabidopsis development. Plant Physiol. 151, 180-198 (2009).

15. Aravind, L. The WWE domain: a common interaction module in protein ubiquitination and ADP ribosylation. Trends Biochem. Sci. 26, 273-275 (2001).

16. Ruf, A., Mennissier De Murcia, J., de Murcia, G. \& Schulz, G. E. Structure of the catalytic fragment of poly(AD-ribose) polymerase from chicken. Proc. Natl Acad. Sci. USA 93, 7481-7485 (1996).

17. Oliver, A. et al. Crystal structure of the catalytic fragment of murine poly (ADPribose) polymerase-2. Nucleic Acids Res. 32, 456-464 (2004).

18. Sousa, F. et al. PARPs and the DNA damage response. Carcinogenesis 33 1433-1440 (2012).

19. Marr, M. T. N. TAF4 takes flight. Proc. Natl Acad. Sci. USA 106, 1295-1296 (2009).

20. Wang, M. et al. ERF109 of trifoliate orange (Poncirus trifoliata (L.) Raf.) contributes to cold tolerance by directly regulating expression of Prx 1 involved in antioxidative process. Plant Biotechnol. J. 17, 1316-1332 (2019).

21. Wang, L. et al. Overexpression of $\mathrm{CmSOS1}$ confers waterlogging tolerance in Chrysanthemum. J. Integr. Plant Biol. 62, 1059-1064 (2020).

22. An, H. CONSTANS acts in the phloem to regulate a systemic signal that induces photoperiodic flowering of Arabidopsis. Development 131, 3615-3626 (2014).

23. Liu, S. et al. A wheat SIMILAR TO RCD-ONE gene enhances seedling growth and abiotic stress resistance by modulating redox homeostasis and maintaining genomic integrity. Plant Cell 26, 164-180 (2014).

24. Shapiguzov, A. et al. Arabidopsis RCD1 coordinates chloroplast and mitochondrial functions through interaction with ANAC transcription factors. Elife 8, e43284 (2019).

25. Vainonen, J. P. et al. RCD1-DREB2A interaction in leaf senescence and stress responses in Arabidopsis thaliana. Biochem. J. 442, 573-581 (2012).

26. Hiltscher, H. et al. The radical induced cell death protein 1 (RCD1) supports transcriptional activation of genes for chloroplast antioxidant enzymes. Front. Plant Sci. 5, 475-475 (2014).

27. Böhlenius, $\mathrm{H}$. et al. CO/FT regulatory module controls timing of flowering and seasonal growth cessation in trees. Science 312, 1040-1043 (2006).

28. Fan, C. et al. Conserved CO-FT regulons contribute to the photoperiod flowering control in soybean. BMC Plant Biol. 14, 9-9 (2014).

29. Shim, J. S., Kubota, A. \& Imaizumi, T. Circadian clock and photoperiodic flowering in Arabidopsis: CONSTANS is a hub for signal integration. Plant Physiol. 173, 5-15 (2017).

30. Zhang, B., Wang, L., Zeng, L., Zhang, C. \& Ma, H. Arabidopsis TOE proteins convey a photoperiodic signal to antagonize CONSTANS and regulate flowering time. Genes Dev. 29, 975-987 (2015).

31. Tamura, K., Dudley, J., Nei, M. \& Kumar, S. MEGA4: molecular evolutionary genetics analysis (MEGA) software version 4.0. Mol. Biol. Evol. 24, 1596-1599 (2007). 
32. Schütze, K., Harter, K. \& Chaban, C. Bimolecular fluorescence complementation (BiFC) to study protein-protein interactions in living plant cells. Methods Mol. Biol. 479, 189-202 (2009).

33. Lai, H. \& Chiang, C. Bimolecular fluorescence complementation (BiFC) assay for direct visualization of protein-protein interaction in vivo. Bio-Protocol 3, e935 (2013).

34. Kost, B., Schnorf, M., Potrykus, I. \& Neuhaus, G. Non-destructive detection of firefly luciferase (LUC) activity in single plant cells using a cooled, slow-scan CCD camera and an optimized assay. Plant J. 8, 155-166 (1995).

35. Francisco-Velilla, R., Fernandez-Chamorro, J., Ramajo, J. \& Martinez-Salas, E. The RNA-binding protein Gemin5 binds directly to the ribosome and regulates global translation. FEBS Lett. 44, 8335-8351 (2016).
36. Cheng, Z. et al. Pathogen-secreted proteases activate a novel plant immune pathway. Nature 521, 213-216 (2015).

37. Yoo, S. D., Cho, Y. H. \& Sheen, J. Arabidopsis mesophyll protoplasts: a versatile cell system for transient gene expression analysis. Nat. Protoc. 2, 1565-1572 (2007).

38. Livak, K. J. \& Schmittgen, T. D. Analysis of relative gene expression data using real-time quantitative PCR and the 2(-Delta Delta C(T)) Method. Methods 25, 402-408 (2001).

39. Blanchard, M. G. \& Runkle, E. S. Use of a cyclic high-pressure sodium lamp to inhibit flowering of chrysanthemum and velvet sage. Sci. Hortic. 122, 448-454 (2009). 